\title{
Perceptual and behavioral adjustments after action inhibition
}

\author{
Wladimir Kirsch • Wilfried Kunde
}

Published online: 13 December 2014

(C) Psychonomic Society, Inc. 2014

\begin{abstract}
Inhibiting a motor action typically prompts a more cautious action mode, leaning toward accuracy rather than speed. In the present study, we explored whether action inhibition is also accompanied by changes of visual perception. Our participants performed goal-directed hand movements from a start to a target position and then judged the starttarget distance. On a proportion of the trials, movement execution had to be stopped before the target position was reached. The results of two experiments revealed smaller start-target distance estimates after interrupted than after unrestricted movements. Moreover, movement amplitudes were decreased in movements that followed interrupted ones. In line with the predictions of action-specific accounts of perception, this outcome indicates that subjective perceptual changes might inform us how to plan future actions.
\end{abstract}

Keywords Embodied cognition · Visual perception

A key characteristic of human behavior is its high flexibility. For instance, we are able to inhibit a planned or even already initiated action when necessary. Such response suppression often results in adaptive adjustments of subsequent behavior, as has been demonstrated in several reaction time experiments (see Verbruggen \& Logan, 2008, for a review). In the widely

Electronic supplementary material The online version of this article (doi:10.3758/s13423-014-0792-1) contains supplementary material, which is available to authorized users.

W. Kirsch · W. Kunde

Department of Psychology, University of Würzburg,

Würzburg, Germany

W. Kirsch $(\triangle)$

Institut für Psychologie III, Universität Würzburg,

Röntgenring 11, D-97070 Würzburg, Germany

e-mail: kirsch@psychologie.uni-wuerzburg.de used stop-signal paradigm (e.g., Logan \& Cowan, 1984), participants typically perform a visual discrimination task that requires a speeded response ("go trials"). On some trials, an auditory stop signal is presented response to which participants are required to withhold their response ("stop trials"). Typically, the responses after previous stop trials are slower than those after previous go trials (e.g., Verbruggen, Logan, Liefooghe, \& Vandierendonck, 2008). Thus, after a stop trial, participants strategically slow down their go responses in order to increase the probability of potential stopping (see, e.g., Bissett \& Logan, 2011, 2012).

In the present article, we explored whether requirements to stop ongoing actions are accompanied by changes of perception. Such changes are suggested by action-specific accounts of perception (see Proffitt, 2008; Proffitt \& Linkenauger, 2013; Witt, 2011a, for reviews). These accounts hold that perception is based upon a reference of initial optical information to motor variables that are relevant for intended actions. Accordingly, changes in motor variables can give rise to changes in visual perception in spite of a constant stimulus. Using a tool that extends one's reaching ability, for example, has proved to decrease the egocentric distance to a target object (Witt, 2011b; Witt \& Proffitt, 2008; Witt, Proffitt, \& Epstein, 2005). Of a particular importance for the present study is the claim that (action-specific) perception prepares the perceiver for a subsequent action by signaling the opportunities and costs associated with that action (e.g., Proffitt, 2006; Witt, 2011a). For example, encumbering a person with a heavy backpack has been reported to make hills look steeper. This change of perception might help one to select an appropriate speed of walking and to avoid excessive demands. Likewise, seeing a target as smaller after an unsuccessful attempt to hit it may help one to exert additional resources in a subsequent attempt. Thus, it is conceivable that cognitive adjustment processes are accompanied by changes of visual perception that facilitate subsequent adaptive behavior. 
Some indirect support for this assumption has come from studies in which researchers have examined the impact of optical illusions on motor behavior (Witt, Linkenauger, \& Proffitt, 2012; Wood, Vine, \& Wilson, 2013). These studies have reported improved performance in a putting task when smaller context stimuli surround a hole than with larger context stimuli. Witt et al. (2012) suggested that if the target appears bigger (in the case of small-context stimuli), the actor improves performance because he or she might expect to be more able to hit the target (which appears easier to hit). These results indicate that changes in perception can be associated with, and can possibly cause, behavioral adjustments.

We explored this issue using a stop-signal task. Participants repeatedly performed a hand movement aimed at a target and subsequently judged a given target distance. On one half of the trials, a stop signal was presented during the movement. In response to this signal, the ongoing movement had to be interrupted. As in the classical stop-signal paradigm, movement interruption can be assumed to entail adjustments in the planning and control of subsequent movements (see also, e.g., van Beers, 2009). In particular, after a stop trial, participants should be more cautious during planning and control of the next movement, in order to increase the chances of potential stopping. This can be achieved through a decrease of applied forces (e.g., of peak force or of the rate of force development). Thus, the parameters of a given movement can be expected to vary, depending on whether or not the previous movement was stopped. The primary question of interest was whether these performance adjustments are preceded by perceptual changes that come with such an adaptive behavior.

In previous studies, we have shown that the parameters of planned movements, such as amplitude, direction, and force, in fact affect visual perception (Kirsch, Herbort, Butz, \& Kunde, 2012; Kirsch \& Kunde, 2013a, 2013b, 2014). Planning a movement of large (as compared with small) amplitude, for example, increases the perceived target distance (see, e.g., Kirsch et al. 2012). These results thus indicate that when changes in planning of an action occur, changes in the visual perception of action-relevant objects can be expected. Accordingly, if planning a movement following movement interruption in the present setup were to differ from planning a movement following an unrestricted movement, then differences in perception after interrupted versus unrestricted movements could emerge. In particular, planning a movement associated with a smaller force impulse following movement interruption is expected to decrease the distance estimate, as compared with planning a movement with a larger force impulse following an unrestricted movement.

In other words, movement interruption might cause strategic adjustments of the initial movement plan, resulting in a tendency to slow down the subsequent movement. During target perception measured after a movement interruption, this adjusted motor plan could then be used as a reference for optical information. ${ }^{1}$ Accordingly, the apparent target distance should decrease following stop trials, as compared with after trials with unrestricted movement. This would correspond to the signal function ascribed to the perception. Because a decrease in target distance typically prompts movements with smaller amplitudes and initial forces (e.g., Gordon \& Ghez, 1987; Messier \& Kalaska, 1999), a decrease in perceived distance after movement interruption would lead to more cautious behavior.

In a few previous studies, we have observed some preliminary evidence for this assumption. When blindfolded participants were asked to move a handle rapidly until it was mechanically stopped and to reproduce that stop position through another unrestricted movement, they tended to slow down the reproduction movement when the initial movement was interrupted shortly after its onset (see Kirsch, Hennighausen, \& Rösler, 2010, Table 1). Interestingly, when the task required a verbal estimate of the movement distance before reproduction, participants substantially underestimated the distance in those conditions (i.e., when movements were stopped shortly after their onsets; Kirsch, Hennighausen, \& Rösler, 2009). Thus, a decrease in subjective distance (after movement interruption) appeared to precede a slower (reproduction) movement.

\section{Method}

\section{Participants}

A group of 24 participants were recruited for Experiment A $\left(M_{\text {age }}=24\right.$ years, $S D=8 ; 20$ female, four male $)$, and 24 participants were recruited for Experiment B $\left(M_{\mathrm{age}}=27\right.$ years, $S D=9 ; 18$ female, six male). All of them reported being righthanders. They gave their informed consent for the procedures and received an honorarium or course credit for their participation.

\section{Apparatus}

The main apparatus included a graphics tablet, a digitizing stylus, a monitor, and a semisilvered mirror (see Fig. 1). The tablet (Intuos $4 \mathrm{XL}$, Wacom) was placed on a table. Above the tablet, a monitor was mounted. The distance between the monitor and the tablet was approximately $47 \mathrm{~cm}$. In the middle, between the monitor and the tablet, was a semisilvered mirror. This apparatus allowed for projections of virtual images on the plane of the tablet, whereas the mirror prevented participants from viewing their arm in a dimmed

\footnotetext{
${ }^{1}$ Such a sensory-motor coupling can be mediated by a process of motor simulation, as was suggested by Witt and Proffitt (2008).
} 
Table 1 Mean distance judgments in Experiments A and B (in millimeters)

\begin{tabular}{|c|c|c|c|c|c|c|c|c|}
\hline & \multicolumn{2}{|l|}{$98.8 \mathrm{~mm}$} & \multicolumn{2}{|l|}{$102.6 \mathrm{~mm}$} & \multicolumn{2}{|l|}{$106.4 \mathrm{~mm}$} & \multicolumn{2}{|l|}{$110.2 \mathrm{~mm}$} \\
\hline & No Stop & Stop & No Stop & Stop & No Stop & Stop & No Stop & Stop \\
\hline \multicolumn{9}{|l|}{ Exp. A } \\
\hline Slow & $106.3(5.9)$ & $105.5(6.3)$ & $109.1(6.6)$ & $108.6(6.6)$ & $113.3(7.6)$ & $112.2(8.0)$ & $116.2(7.3)$ & $115.6(8.3)$ \\
\hline Fast & $108.4(7.6)$ & $109.0(6.6)$ & $113.3(7.0)$ & $112.8(7.5)$ & $116.3(8.1)$ & $114.4(7.5)$ & $121.2(8.3)$ & $120.1(9.2)$ \\
\hline \multicolumn{9}{|l|}{ Exp. B } \\
\hline Slow & $111.5(6.6)$ & $110.5(5.7)$ & $114.8(6.4)$ & $113.7(6.7)$ & $118.6(7.1)$ & $117.6(6.5)$ & $122.4(7.0)$ & $121.8(7.7)$ \\
\hline Fast & $110.8(7.0)$ & $111.0(6.4)$ & $113.9(7.4)$ & $114.0(6.6)$ & $118.2(8.1)$ & $117.9(7.5)$ & $122.6(7.0)$ & $121.6(7.6)$ \\
\hline
\end{tabular}

Standard deviations are in parentheses

lab. One pixel (px) of the monitor was approximately $0.38 \mathrm{~mm}$ in size. Headphones were used to present acoustic signals (see below). The participants sat in front of the apparatus with the middle of their body corresponding with the middles of both the monitor and the tablet. They were asked to lean their forehead on an upper part of the apparatus. Stylus movements had to be performed with the right hand. Perceptual adjustments, in contrast, were made with the left hand.

\section{Procedure}

The basic procedure included stylus movements from a start to a target position (gray dots, $\sim 2 \mathrm{~mm}$ in size) and subsequent estimations of the start-target distance by a method of adjustment. Besides the movement stopping, we varied movement velocity and the time of the backward movement. Under certain conditions, participants' responses were considered errors. In the following sections, we describe each of these aspects of the procedure in more detail.

Target movement The target movement was performed in the absence of visual feedback. ${ }^{2}$ On $50 \%$ of trials, an acoustic signal (a sequence of short beep tones, $2000 \mathrm{~Hz}$ ) was presented during the movement (stop trials). In response to this signal, participants had to immediately stop the movement and press a stylus button. Pressing this button turned the sound off. On the remaining trials, the movement was unrestricted (no-stop trials). Irrespective of whether or not the movement was stopped, participants were instructed to judge the visual start-to-target distance (not the distance that they had effectively covered by their hand).

Distance estimates Distance estimates were measured by means of an additional dot presented to the left of the start position (initially at a distance corresponding to either $50 \%$ or

\footnotetext{
2 The stylus position was only displayed during the backward movement (top dot in Fig. 1).
}

$150 \%$ of the start-target distance). The task here was to adjust the position of this additional dot so that the "horizontal" distance was equal to the distance between the start and target dots. This was done by pressing the left and right arrow keys on a keyboard. The distance estimate had to be confirmed by the Enter key.

Using this method in the present context was based on the assumption of a dimensional overlap (Kornblum, Hasbroucq, \& Osman, 1990) between motor and perceptual processes in the case of the dot that served as target, but not in case of the dot that served as a comparison circle. In other words, we assumed that changes in motor processes are accompanied by changes in the perception of objects that are relevant for the intended actions (see, e.g., Kirsch \& Kunde, 2013b; Witt et al. 2005). Because only the target dot was relevant for movement planning in the present setup, perception of the comparison dot was assumed to be unaffected by the critical manipulation of movement interruption. Thus, any changes in the distance estimates were assumed to emerge from changes in the perception of the target dot. $^{3}$

Velocity instruction The main question of interest was whether and how movement interruptions would change distance estimates. In our previous experiments using mechanical movement interruptions, the speed of the movement proved to have an impact on judgment behavior. These preliminary results indicated a tendency to underestimate the target distance in restricted (as compared with unrestricted) movements when the movement speed was rather fast, and to overestimate the distance when the movement speed was rather low. We speculated that such perceptual biases might be related to adjustments of subsequent actions (such as the speeding up and slowing down of subsequent movements). We thus included a velocity variation in the present study in order to examine whether a possible perceptual bias was affected by

\footnotetext{
${ }_{3}^{3}$ This method and similar ones have often been applied by us and others in related contexts (e.g., Kirsch \& Kunde, 2013a, b; Witt \& Proffitt, 2008; Witt et al. 2005).
} 


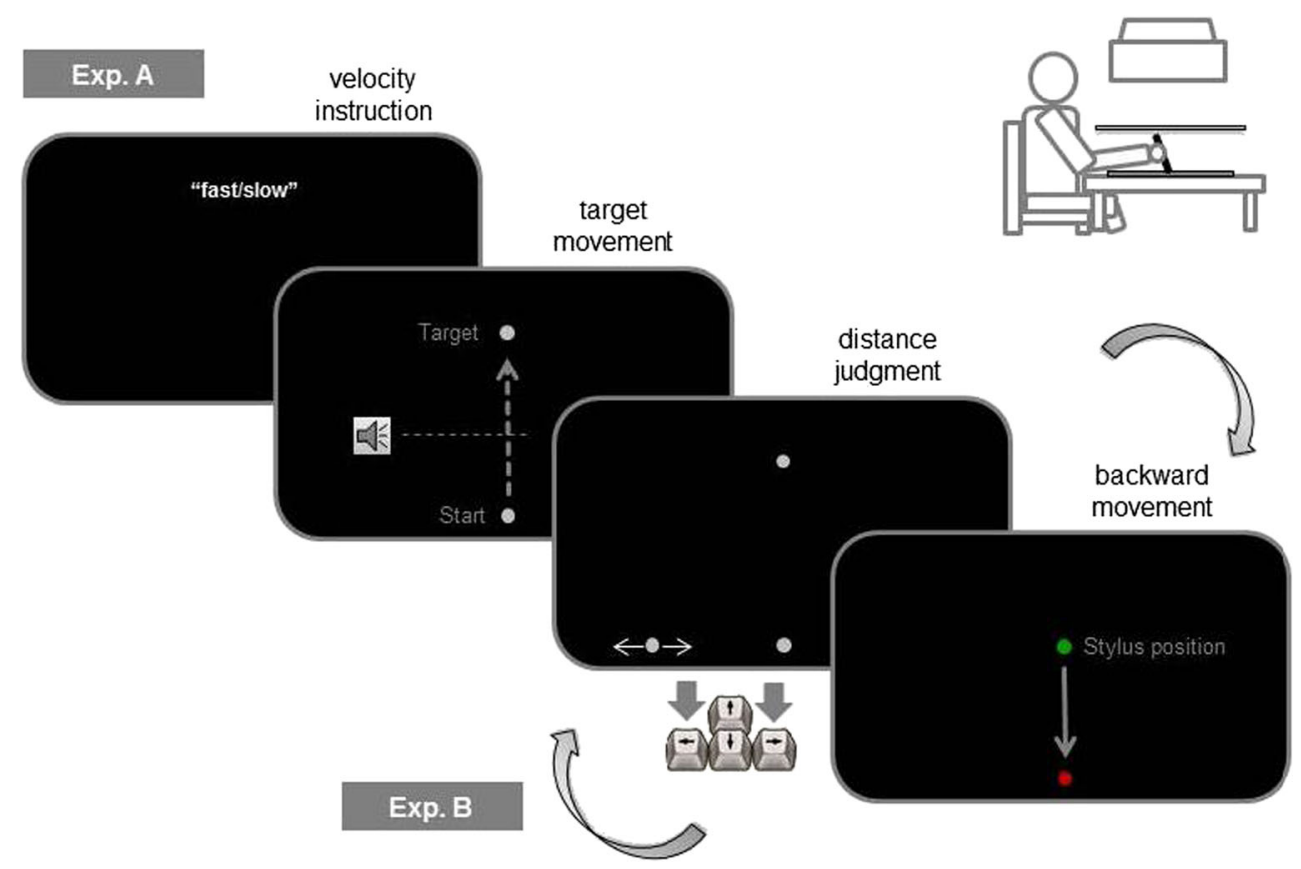

Fig. 1 Main trial events and the apparatus (upper right corner) of the present study. After the velocity instruction appeared, participants had to press the space bar. Then the stylus had to be moved from the start to the target position. On $50 \%$ of trials, a stop signal was presented during the movement. In Experiment A, a distance estimate was done immediately

movement speed when movement was voluntarily stopped. For instance, the predicted decrease of distance estimates following movement stopping might be smaller for slow movements than for fast movements (or might even result in an increase of estimates). Accordingly, half of the participants were asked to perform fast movement, whereas the other participants were asked to move the stylus slowly (random assignment). This velocity instruction was also shown at the beginning of each trial, to encourage participants to keep it in mind in the course of the experiment (see Fig. 1).

Time of the backward movement Figure 1 outlines the main trial procedures of Experiment A and Experiment B. In an initial experiment (Exp.A), the distance judgment was made immediately after movement interruption - that is, before the hand was returned to the start position. In our earlier studies, we had demonstrated that an increase in the planned movement amplitude increases distance estimates (e.g., Kirsch et al. 2012; see also the introduction). Because the amplitude of the backward movement was larger for unrestricted than for restricted movements in the setup of Experiment A, the predicted decrease in distance estimates for restricted movements might have been due to the planning of the backward movement rather than to the movement interruption per se. We thus conducted an additional experiment (Exp. B) to control for this possible confound. In this second experiment, we basically interchanged the order of the distance judgment and the backward movement. after the target movement was completed (i.e., before the backward movement). In Experiment B, the stylus was moved back to the start before the distance judgment. Note that the stylus position was visible only during the backward movement

Error feedback Under the following conditions, error feedback was presented and the current trial was repeated: when the stylus button was pressed before the stop position was achieved or when the distance between the end position of the stylus and the target fell below $19 \mathrm{~mm}$ in the stop trials ${ }^{4}$; when the end position of the stylus deviated by more than $19 \mathrm{~mm}$ from the target after the movement in the no-stop trials; when the distance judgment was confirmed without moving the horizontal dot; or when the stylus was moved away from the start position during the distance judgment (and during the velocity instruction in Exp. B). On average, $14 \%$ of the trials were repeated in Experiment A, and 19\% in Experiment B.

\section{Design}

The start position was always constant during the experiment, whereas the position of the target varied slightly trial by trial. For slow movements, the stop signal was presented after half of the target distance (related to $y$-coordinates) had been covered by the stylus. In order to make the end positions comparable across the slow and fast conditions, the stop signal was presented much earlier during the fast movements. Depending on the current target distance, the signal was presented after the participant had exceeded $11.5 \%, 13.0 \%, 14.3 \%$, or $15.5 \%$ of the target distance (see below). This was done in light of the

\footnotetext{
${ }^{4}$ This ensured that movements were stopped after the stop signal and before the target was reached.
} 
results of pilot experiments indicating a considerably larger movement extent following the stop signal for fast than for slow movements. As is shown in Fig. S1 (see the supplemental materials), the end positions of the movements in the present study were comparable for the slow and fast conditions. Thus, the variation in the timing of the stop signal was successful.

We used four target distances $(98.8,102.6,106.4$, and $110.2 \mathrm{~mm}$ ), two stop conditions (stop and no stop), and two velocity instructions (fast and slow, which were varied between participants; see above). Each experiment (i.e., Exp. A and Exp.B) was divided into two blocks that included 40 trials each. In each block, each combination of target distance and stop condition was presented five times in a randomized order. At the beginning of each experiment, participants performed 16 practice trials, which were not included in the analyses.

\section{Data analysis}

Trials in which the distance estimates were below or above 2 $S D$ s of the median - as computed for each participant, each target distance, and each stop condition - were excluded from the analysis. Overall, 96.8\% (Exp.A) and 97.6\% (Exp.B) of trials were entered into the analyses.

\section{Results}

Table 1 provides an overview of the mean distance estimates across all conditions.

An analysis of variance (ANOVA) including Target Distance and Stop Condition as within-participants factors and Velocity Instruction as a between-participants factor revealed significant main effects of target distance and stop condition for Exp. A [respectively, $F(3,66)=193.7, p<.001$, $\eta_{\mathrm{p}}{ }^{2}=.898$, and $\left.F(1,22)=5.4, p=.029, \eta_{\mathrm{p}}{ }^{2}=.198\right]$, as well as for Exp. B [respectively, $F(3,66)=260.2, p<.001, \eta_{\mathrm{p}}{ }^{2}=$ .922 , and $\left.F(1,22)=5.2, p=.032, \eta_{\mathrm{p}}{ }^{2}=.192\right]$. Neither velocity instruction nor the interactions reached the significance threshold (all $p \mathrm{~s}>.064){ }^{5}$

Judgments increased with distance, as expected (see Table 1). More importantly, after movement interruption, participants decreased their estimates as compared with judgments made after unrestricted movements (see the left panels of Fig. 2 for the means). This rather small but systematic effect was present in both experiments. Unlike in our previous experiments using mechanical movement interruptions, the speed of the movement proved to have no systematic impact on judgment behavior.

\footnotetext{
5 This marginally significant $p$ value corresponded to the interaction between target distance and velocity instruction in Exp. A and suggested a distance-specific trend toward larger distance estimates for the participants getting the fast-movement instruction (see Table 1; all other $p$ values $>.22$ ).
}

We also examined how movement interruption affected motor behavior in the subsequent trial. Unlike in the classical stop-signal paradigm, the temporal aspects of the responses in the more complex task of the present study were not rigorously controlled. In particular, movement trajectories were not recorded, and there was no speed instruction for pressing the stylus button after a movement had been completed (or stopped). Thus, we had no reliable measure of movement time (or of stop-signal reaction times), but instead focused on movement amplitude, which is an indicator of the motor commands used to reach a given target.

For stop trials, the amplitude indicates how cautious the participants are during movement execution (i.e., how fast they are and/or how much force they apply). Fast movements are usually more difficult to stop than slow movements (see also, e.g., Bissett \& Logan, 2011). In the present study, for example, the stop signal was presented much earlier for fast than for slow movements, as we mentioned earlier (at about $14 \%$ vs. $50 \%$ of the trajectory). In spite of this, the movement amplitudes were similar for both velocity conditions (see Fig. S1). Accordingly, an increase of movement speed and/ or force during the stop trials must be associated with an increase in difficulty to stop the movement. As a consequence, an increase in the amplitude of a movement finished in response to a stop signal will indicate a decrease of caution in planning and control strategies (i.e., an increase of speed and/ or force).

For no-stop trials, in contrast, the movement amplitude informs us about the current sensorimotor mapping. In the absence of the stop signal, the task was to reach the current target position. Because no visual feedback was provided until the movement was finished, the end position of the movement (i.e., movement amplitude) can be considered indicative of the planned movement extent required to achieve a given target (i.e., of the current calibration between motor and visual representations).

Here, we defined movement amplitude as the linear distance between the start position and the end position of the movement along the $y$-axis of the tablet. The amplitude of the movement performed after stop trials was compared with the amplitude of the movement performed after no-stop trials by means of an ANOVA including Stop Condition in a Given Trial $n$ and Stop Condition in Trial $n-1$ as factors. This analysis revealed significant main effects of the stop conditions in trial $n-1$ of both Experiments $\mathrm{A}$ and $\mathrm{B}$, with $F(1,23)=33.6, p<.001, \eta_{\mathrm{p}}{ }^{2}=.593$, and $F(1,23)=9.4, p=$ $.005, \eta_{\mathrm{p}}{ }^{2}=.291$, respectively. This effect was due to smaller movement amplitudes after stop trials than after no-stop trials (see Fig. 2, right panels). Pairwise comparisons suggested further that this effect held for restricted as well as for unrestricted movements on trial $n[t(23)=3.9, p=.001$, and $t(23)=$ $4.5, p<.001$, respectively, for Exp. A, and $t(23)=2.1, p=$ .052 , and $t(23)=2.3, p=.033$, for Exp.B]. 


\section{Experiment $\mathrm{A}$}

distance judgment

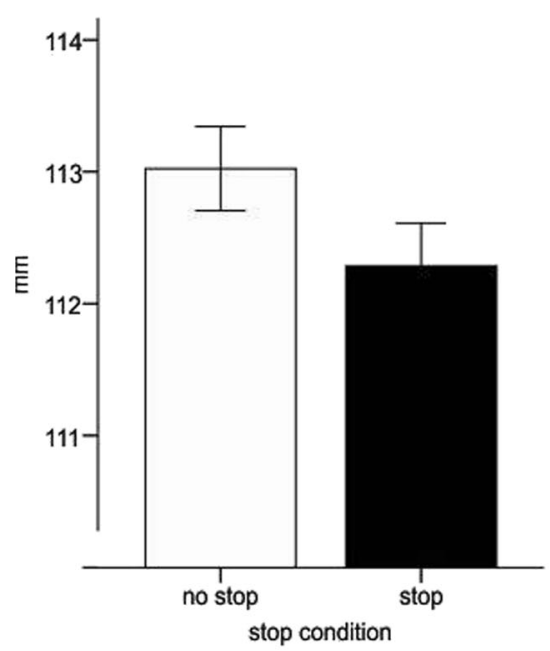

distance judgment

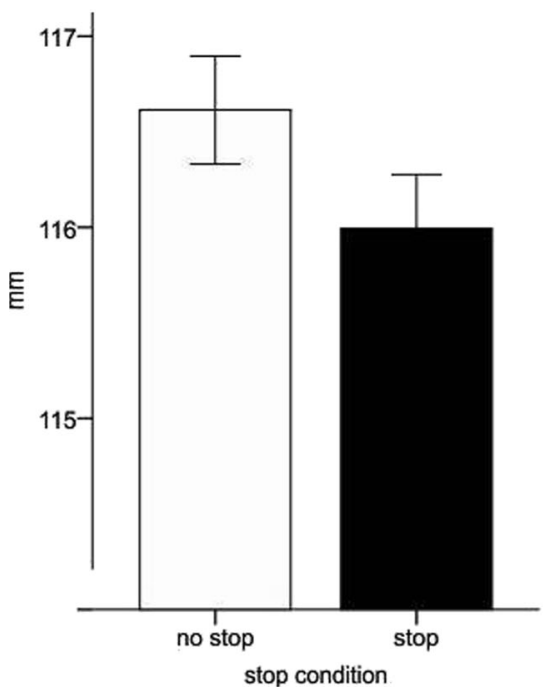

Fig. 2 Main results of Experiment A (top) and Experiment B (bottom). Left panels: Mean distance estimates for each stop condition. Right panels: Mean movement amplitudes, depending on whether the previous

\section{Discussion}

We asked whether the cognitive adjustment processes associated with inhibition of goal-directed movements are accompanied by changes in apparent target distance. Participants performed hand movements that initially were attempting to reach a target. These movements either were interrupted by a stop signal before the target was achieved or were unrestricted. A decrease of the perceived distance after interrupted as compared with unrestricted movements was predicted, due to an assumed functional role of perception for action: After movement interruption, perception might signal to the actor to movement amplitude

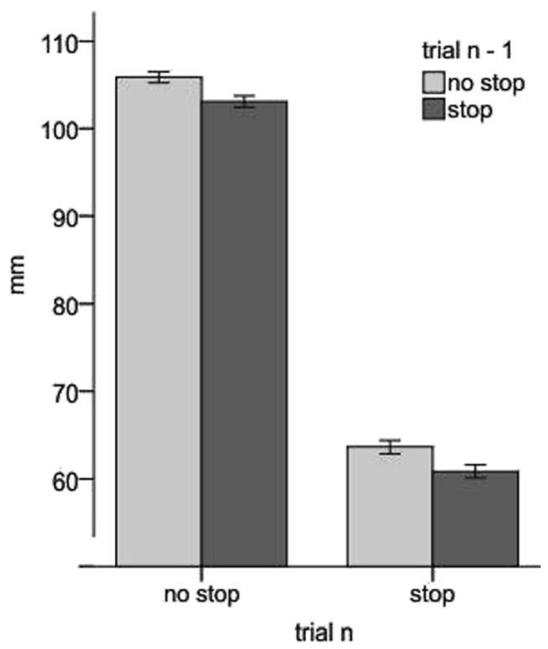

\section{Experiment B}

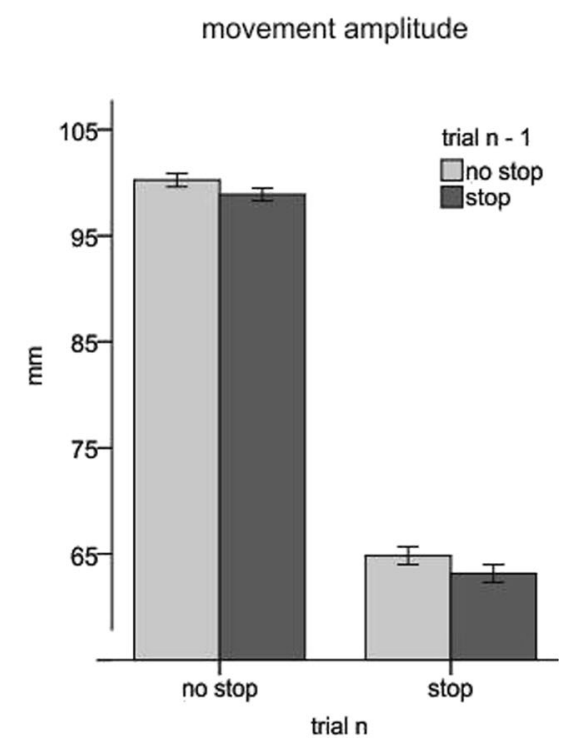

trial $(n-1)$ had been from the same or a different stop condition. Note that error bars reflect within-participants confidence intervals (95\%) computed according to Cousineau (2005)

be more cautious during a subsequent action in the present setup. The results were in line with this prediction.

Distance judgments following interrupted movements decreased as compared with those following unrestricted movements. Moreover, we found that the movement amplitude in a current trial was affected by the stop condition in the previous trial: That is, the amplitude was smaller following movement interruption than following unrestricted movements. This last result suggests that participants performed movements with more caution after stop trials, as expected. This was indicated by shorter amplitudes in stop trials following other stop trials. Moreover, the shorter amplitudes following stop trials that we 
observed in no-stop trials might also indicate a changed mapping between the visual stimuli and movements. In particular, a smaller movement extent might have been associated with a given visual distance after restricted than after unrestricted movements. This would indicate that movement interruption induced a short-term recalibration between the optical information associated with the target position and the motor command required to achieve this position. Also, this finding is in line with changes in visual perception observed in the preceding trial and with their assumed (signal) function. In other words, the smaller movement amplitudes here might be a consequence of perceiving the previous target as being closer.

Thus, the results as a whole seem to delineate a type of visual illusion that may help optimize the planning of future actions: This illusion appears to inform people about how to move, by means of a subjective distortion. We assume that the underlying mechanism is related to strategic adjustments of the motor plan used to achieve a given visual target. In particular, movement interruption indicates to the participant that the motor output is inappropriate. This conflict will be resolved by updating the initial motor plan. According to the core assumption of action-specific accounts of perception, visual stimuli are perceived in terms of motor units (e.g., Proffitt \& Linkenauger, 2013). Thus, changes in the motor plan may cause changes in perception (see also the introduction).

Such a mechanism resembles to some extent the "conflictinduced perceptual filtering" suggested earlier (Wendt, LunaRodriguez, \& Jacobsen, 2012). According to this account, the perceptual system can be adjusted depending on the degree of cognitive conflict induced, for example, by the Eriksen flanker task (Eriksen \& Eriksen, 1974). For instance, an increase in the congruent/incongruent trial ratio might enhance the efficiency of processing the stimuli serving as flankers and/or might reduce the efficiency of processing the stimuli serving as targets. Thus, perceptual processing is assumed to change as a result of the attempt to resolve a cognitive conflict ${ }^{6}$ (see also Egner \& Hirsch, 2005).

So far, we have assumed that strategic performance adjustments are the primary cause of the perceptual changes observed in the present setup. This assumption seems justified, given the evidence for strategic post-stop-signal adjustments within the stop-signal paradigm (e.g., Bissett \& Logan, 2012; Verbruggen \& Logan, 2009). Nevertheless, other explanations are possible. For example, a decrease in distance estimates following movement interruption (as well as variations in the subsequent movements) might be an aftereffect of motor

\footnotetext{
${ }^{6}$ This hypothesis is rooted in the conflict-monitoring model of Botvinick, Braver, Barch, Carter, and Cohen (2001), which assumes attentional biasing of perceptual processes as a function of the degree of the previous conflict. Our conclusions are thus also compatible with this model.
}

inhibition, rather than directly related to behavioral control adjustments (see, e.g., Verbruggen et al., 2008). That is, breaking an ongoing movement could per se decrease the perceived target distance. For instance, distance perception can be modified when the uncertainty (i.e., noise) within the sensorimotor system increases (Bourgeois \& Coello, 2012). Accordingly, the observed decrease of distance judgments for restricted movements might result from increased sensorimotor variability due to the movement interruption, rather than from processes associated with performance adjustments, as we have suggested. The present study cannot distinguish between these possible mechanisms, and further research will be needed to examine the exact origin of the observed effects.

To sum up, the present results show-to our knowledge, for the first time - that an action-related change in visual perception is followed by adjustments of successive behavior that are in line with a changed perception and that are consistent with a strategic adaptation to the given task demands. However, further studies will be needed to better evaluate the extent to which the relation between changes in perception and successive behavior is causal.

Author note This research was supported by grant KI 1620/1-1 awarded to W. Kirsch by the German Research Council (DFG).

\section{References}

Bissett, P. G., \& Logan, G. D. (2011). Balancing cognitive demands: Control adjustments in the stop-signal paradigm. Journal of Experimental Psychology: Learning, Memory, and Cognition, 37, 392-404. doi:10.1037/a0021800

Bissett, P. G., \& Logan, G. D. (2012). Post-stop-signal slowing: Strategies dominate reflexes and implicit learning. Journal of Experimental Psychology: Human Perception and Performance, 38, 746-757. doi:10.1037/a0025429

Botvinick, M. M., Braver, T. S., Barch, D. M., Carter, C. S., \& Cohen, J. D. (2001). Conflict monitoring and cognitive control. Psychological Review, 108, 624-652. doi:10.1037/0033-295X.108.3.624

Bourgeois, J., \& Coello, Y. (2012). Effect of visuomotor calibration and uncertainty on the perception of peripersonal space. Attention, Perception, \& Psychophysics, 74, 1268-1283. doi:10.3758/ s13414-012-0316-x

Cousineau, D. (2005). Confidence intervals in within-subject designs: A simpler solution to Loftus and Masson's method. Tutorials in Quantitative Methods for Psychology, 1, 75-78.

Egner, T., \& Hirsch, J. (2005). Cognitive control mechanisms resolve conflict through cortical amplification of task-relevant information. Nature Neuroscience, 8, 1784-1790. doi:10.1038/nn1594

Eriksen, B. A., \& Eriksen, C. W. (1974). Effects of noise letters upon the identification of a target letter in a nonsearch task. Perception \& Psychophysics, 16, 143-149. doi:10.3758/BF03203267

Gordon, J., \& Ghez, C. (1987). Trajectory control in targeted force impulses: II. Pulse height control. Experimental Brain Research, 67, 241-252. doi:10.1007/BF00248546

Kirsch, W., Hennighausen, E., \& Rösler, F. (2009). Dissociating cognitive and motor interference effects on kinesthetic short-term memory. Psychological Research, 73, 380-389. doi:10.1007/s00426-0080159-1 
Kirsch, W., Hennighausen, E., \& Rösler, F. (2010). ERP correlates of linear hand movements in a motor reproduction task. Psychophysiology, 47, 486-500. doi:10.1111/j.1469-8986.2009. 00952.x

Kirsch, W., Herbort, O., Butz, M. V., \& Kunde, W. (2012). Influence of motor planning on distance perception within the peripersonal space. PLoS ONE, 7, e34880. doi:10.1371/journal. pone. 0034880

Kirsch, W., \& Kunde, W. (2013a). Moving further moves things further away in visual perception: Position-based movement planning affects distance judgments. Experimental Brain Research, 226, 431440. doi:10.1007/s00221-013-3455-y

Kirsch, W., \& Kunde, W. (2013b). Visual near space is scaled to parameters of current action plans. Journal of Experimental Psychology: Human Perception and Performance, 39, 1313-1325. doi:10.1037/ a0031074

Kirsch, W., \& Kunde, W. (2014). Impact of planned movement direction on judgments of visual locations. Psychological Research, 78, 705720. doi:10.1007/s00426-013-0512-x

Kornblum, S., Hasbroucq, T., \& Osman, A. (1990). Dimensional overlap: Cognitive basis for stimulus-response compatibility-A model and taxonomy. Psychological Review, 97, 253-270. doi:10.1037/0033295X.97.2.253

Logan, G. D., \& Cowan, W. B. (1984). On the ability to inhibit thought and action: A theory of an act of control. Psychological Review, 91, 295-327. doi:10.1037/0033-295X.91.3.295

Messier, J., \& Kalaska, J. F. (1999). Comparison of variability of initial kinematics and endpoints of reaching movements. Experimental Brain Research, 125, 139-152. doi:10.1007/s002210050669

Proffitt, D. R. (2006). Embodied perception and the economy of action. Perspectives on Psychological Science, 1, 110-122. doi:10.1111/j. 1745-6916.2006.00008.x

Proffitt, D. R. (2008). An action-specific approach to spatial perception. In R. L. Klatzky, B. MacWhinney, \& M. Behrmann (Eds.), Embodiment, ego-space, and action (pp. 177-200). New York, NY: Psychology Press.

Proffitt, D. R., \& Linkenauger, S. A. (2013). Perception viewed as a phenotypic expression. In W. Prinz, M. Beisert, \& A. Herwig (Eds.), Action science: Foundations of an emerging discipline (pp. 171198). Cambridge, MA: MIT Press. van Beers, R. J. (2009). Motor learning is optimally tuned to the properties of motor noise. Neuron, 63, 406-417. doi:10.1016/j.neuron. 2009.06.025

Verbruggen, F., \& Logan, G. D. (2008). Response inhibition in the stopsignal paradigm. Trends in Cognitive Sciences, 12, 418-424. doi:10. 1016/j.tics.2008.07.005

Verbruggen, F., \& Logan, G. D. (2009). Proactive adjustments of response strategies in the stop-signal paradigm. Journal of Experimental Psychology: Human Perception and Performance, 35, 835-854. doi:10.1037/a0012726

Verbruggen, F., Logan, G. D., Liefooghe, B., \& Vandierendonck, A. (2008). Short-term aftereffects of response inhibition: Repetition priming or between-trial control adjustments? Journal of Experimental Psychology: Human Perception and Performance, 34, 413-426. doi:10.1037/0096-1523.34.2.413

Wendt, M., Luna-Rodriguez, A., \& Jacobsen, T. (2012). Conflict-induced perceptual filtering. Journal of Experimental Psychology: Human Perception and Performance, 38, 675-686.

Witt, J. K. (2011a). Action's effect on perception. Current Directions in Psychological Science, 20, 201-206. doi:10. 1177/0963721411408770

Witt, J. K. (2011b). Tool use influences perceived shape and perceived parallelism, which serve as indirect measures of perceived distance. Journal of Experimental Psychology: Human Perception and Performance, 37, 1148-1156. doi:10.1037/a0021933

Witt, J. K., Linkenauger, S. A., \& Proffitt, D. R. (2012). Get me out of this slump! Visual illusions improve sports performance. Psychological Science, 23, 397-399. doi:10.1177/0956797611428810

Witt, J. K., \& Proffitt, D. R. (2008). Action-specific influences on distance perception: A role for motor simulation. Journal of Experimental Psychology: Human Perception and Performance, 34, 1479-1492. doi:10.1037/a0010781

Witt, J. K., Proffitt, D. R., \& Epstein, W. (2005). Tool use affects perceived distance but only when you intend to use it. Journal of Experimental Psychology: Human Perception and Performance, 31, 880-888. doi:10.1037/0096-1523.31.5.880

Wood, G., Vine, S. J., \& Wilson, M. R. (2013). The impact of visual illusions on perception, action planning, and motor performance. Attention, Perception, \& Psychophysics, 75, 830-834. doi:10.3758/ s13414-013-0489-y 